\title{
Kündig's experiment on the transverse Doppler shift re-analyzed
}

\author{
A L Kholmetskii ${ }^{1}$, T Yarman ${ }^{2}$ and O V Missevitch ${ }^{3}$ \\ ${ }^{1}$ Department of Physics, Belarusian State University, 4, Nezavisimosti Avenue, 220050 Minsk, Belarus \\ ${ }^{2}$ Department of Engineering, Okan University Istanbul, Turkey and Savronik, Eskisehir, Turkey \\ ${ }^{3}$ Institute for Nuclear Problems, Belarus State University, 220030 Minsk, Belarus \\ E-mail:kholm@bsu.by
}

Received 5 November 2007

Accepted for publication 4 January 2008

Published 6 February 2008

Online at stacks.iop.org/PhysScr/77/035302

\begin{abstract}
In this paper, we re-analyze the ingenious experiment by Kündig (measurement of the transverse Doppler shift by means of the Mössbauer effect) and show that a correct processing of experimental data gives a relative energy shift $\Delta E / E$ of the absorption line different from the value of classically assumed relativistic time dilation for a rotating resonant absorber. Namely, instead of the relative energy shift $\Delta E / E=-(1.0065 \pm 0.011) v^{2} / 2 c^{2}$ reported by Kündig ( $v$ being the linear velocity of absorber and $c$ being the light velocity in vacuum), we derive from his results $\Delta E / E=-(1.192 \pm 0.011) v^{2} / 2 c^{2}$. We are inclined to think that the revealed deviation of $\Delta E / E$ from relativistic prediction cannot be explained by any instrumental error and thus represents a physical effect. In particular, we assume that the energy shift of the absorption resonant line is induced not only by the standard time dilation effect, but also by some additional effect missed at the moment, and related perhaps to the fact that resonant nuclei in the rotating absorber represent a macroscopic quantum system and cannot be considered as freely moving particles.
\end{abstract}

PACS numbers: 03.30.+p, 76.80.+y

\section{Introduction}

Nowadays, the basic physics of the relativistic time dilation has been verified to a high degree of accuracy. Historically, the dilation of time for moving objects was first verified experimentally in [1]. The quantitative measurements of the time dilation effect have been carried out in a series of Mössbauer experiments in rotating systems [2-7]. Later, much more precise experiments with ion beams confirmed this relativistic effect with the accuracy of about $10^{-9}([8,9]$ and references therein) and left no room for any doubts about its validity. At the same time, one should emphasize that in the experiments mentioned above, the effect of time dilation was verified, in fact, for essentially different physical conditions: charged particles in an ion beam can be considered as moving freely, whereas resonant nuclei in Mössbauer experiments are bound in a solid body and constitute a macroscopic quantum system. Thus, despite a huge difference in measuring precision: $10^{-9}$ for ion beam measurements and $10^{-2}$ for Mössbauer measurements, the latter have their own independent significance for verification of the unified character of dilation of time both for free and bound atoms. Among the known Mössbauer experiments, mentioned above, the experiment by Kündig implemented almost 45 years ago [2] remains the most precise, because Kündig was the one who successfully applied a modulation of energy of resonant radiation in a rotating system. This method allowed measuring a position of the resonant line on the energy scale, which is unambiguously related to the transverse Doppler shift regardless of possible chaotic vibrations in the rotor. This is due to the fact that such vibrations may change the shape of the resonant line, but not its position on the energy scale. Kündig reported an experimental confirmation of the transverse Doppler effect (or, which is the same for his configuration, relativistic dilation of time) with the accuracy of about $1 \%$. The result obtained by Kündig and by the authors of [3-7] deprived physicists of interest in further repeating similar measurements, and, in the last few decades, this experiment was often referred to as one of the remarkable confirmations of the relativity theory. Nevertheless, we re-analyzed the experimental results and revealed some ambiguous points (section 2). In section 3, we consider at a 


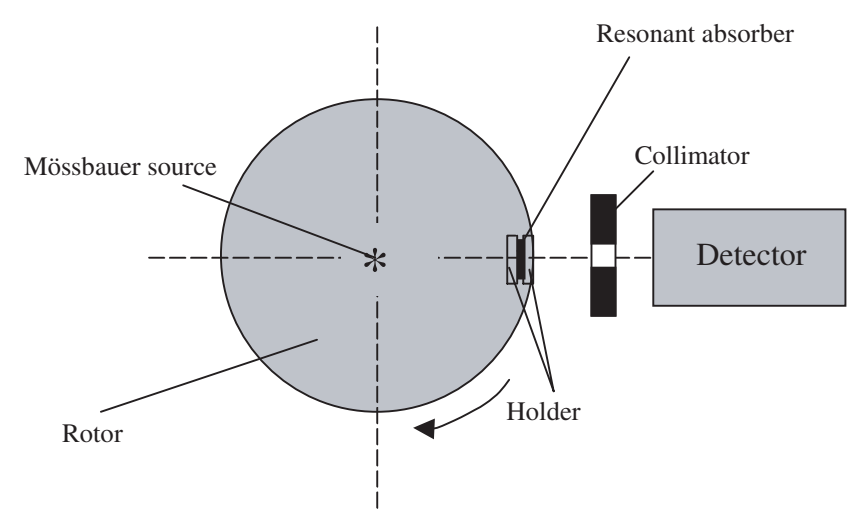

Figure 1. Schematic diagram of Kündig's experiment.

qualitative level similar experiments [3-7], and also find that they are not conclusive. A possible deviation of measured energy shift from the relativistic time dilation value is discussed in section 4. Finally, section 5 contains some conclusions.

\section{Processing of experimental data and comparison with Kündig's results}

In the Kündig experiment, the source of Mössbauer radiation, ${ }^{57} \mathrm{Co}$, plated on $\alpha$-iron was located at the rotational axis of the rotor (figure 1). The latter was machined from a special aluminum alloy and had a diameter of $20 \mathrm{~cm}$. The absorber, a 0.25 -mil-thick foil of $91 \%$ enriched ${ }^{57} \mathrm{Fe}$, was placed inside a $1 / 16$ in.-thick Plexiglas disk and was mounted at a radius of $R_{\mathrm{A}}=9.3 \mathrm{~cm}$. The source and the absorber were mounted in a hole of $1 \mathrm{~cm}$ diameter, which was drilled diametrically through the rotor. The source was glued to an isolating piece of Plexiglas mounted on the face of a piezoelectric transducer. A periodic symmetric triangle voltage signal was applied to the piezotransducer, providing a corresponding triangle law of displacement of the ${ }^{57} \mathrm{Co}$ source and realizing by such a way a constant velocity mode of source oscillation. This ingenious technical method allows establishing a direct proportionality between the amplitude of the reference triangle voltage signal and the value of relative velocity between the source and absorber. A resonant radiation passing through the absorber was detected by two stationary proportional counters beyond the rotor. Technical details of the registration system can be found in the original paper [2]. It is important that when applying different amplitudes $U$ of the reference signal to the piezotransducer under fixed rotor angular frequency $\omega$, the experimenter could directly measure a shape of resonant line versus $U$ for various $\omega$. To complete his measurements, Kündig separately carried out calibration measurements, when the piezotransducer with the attached source was mounted on a mechanical linear drive, and for different known linear velocities $u$ of the drive, the resonant absorption was measured versus $U$ with the same absorber and proportional detector as in the rotor experiment. Then, the measured shift of resonant lines $D$ (in volts) is directly related to a given value of $u$. Further on, applying the least-square method, Kündig obtained the function $D(u)$, which allows determining $D$ in the units of a relative energy shift $\Delta E / E=u / c$. The last step was to recalculate a set of values of $D$, obtained in the rotor experiment, into a relative energy shift of resonant lines as the function of $\omega$. Kündig's processing of experimental data gives the value

$$
\frac{\Delta E}{E}=-(1.0065 \pm 0.011) \frac{R_{\mathrm{A}}^{2} \omega^{2}}{2 c^{2}},
$$

which (according to Kündig's evaluation) perfectly agrees with the relativistic dilation of time on a rotating disk, i.e.

$$
\frac{\Delta E}{E}=\sqrt{1-\frac{\mathrm{R}_{\mathrm{A}}^{2} \omega^{2}}{c^{2}}}-1 \approx-\frac{R_{\mathrm{A}}^{2} \omega^{2}}{2 c^{2}} .
$$

However, in this interesting experiment, the data processing seems questionable.

First we transformed into numerical form the experimentally obtained curves presented in figures 3 and 4 of [2] and carried out independent processing of Kündig's data. We have found that the presented results of a rotor experiment at rotation frequencies 11000,21000 and $31000 \mathrm{rpm}$ (figure 3 of [2]), as well as the calibration data given at $u=0,0.1713$ and $0.3499 \mathrm{~mm} \mathrm{~s}^{-1}$ (figure 4 of [2]), are correct: the numerical data in figures 3 and 4 and the positions of corresponding extremes of drawn curves exactly coincide with each other. Further on, we have applied the same numerical analysis to Kündig's calibration curve, figure 5 of [2]. This curve was approximated by a parabola, and the least-square fit implemented by Kündig gave the numerical coefficients as follows:

$$
D=(0.64 \pm 0.4)+(174.85 \pm 0.38) u-(1.79 \pm 0.85) u^{2} .
$$

However, at least the second coefficient in equation (3) is wrong. In figure 2, we show three experimental points from the calibration measurements of figure 4 of [2] in comparison with the curve (3) (line (a)). One can see that the dependence (3) does not describe the experimental data, which allows us to assume a misprint in presentation of calibration coefficients. In these conditions, we can plot our own calibration curve, using three calibration points, available in figure 4 of [2] at $u=0,0.1713$ and $0.3499 \mathrm{~mm} \mathrm{~s}^{-1}$. Assuming a linear dependence of $D$ on $v$ (i.e. neglecting the very small term with $u^{2}$ ), we obtained, after the least square fit,

$$
D=(0.30 \pm 0.1)+(108.3 \pm 0.70) u .
$$

The dependence (4) is depicted in figure 2 as a bold continuous line (b), and it adequately describes the calibration data. Comparing the dependencies (3) and (4), we point out that the difference of the first terms in their right-hand sides does not essentially influence $D$, but the second coefficients (which are the most crucial) differ by $\sim 70 \%$.

Thus, due to Kündig's misprint, we cannot evaluate the details of his further calculations summarized in the table of [2]. The three left columns of table 1 reproduce the results obtained by Kündig: the second column presents the values of shift $D$ obtained in the rotor experiment for different $\omega$, recalculated with the calibration curve into velocity units; the third column shows the computed ratio $D /\left(R_{\mathrm{A}}^{2}-R_{\mathrm{S}}^{2}\right) \omega^{2}$ ( $R_{\mathrm{S}} \ll R_{\mathrm{A}}$ being an average radial co-ordinate of the source). One can see that the weighted average of this ratio is well matched to the expected value $1 / 2 c=1.688 \times 10^{-9} \mathrm{~s} \mathrm{~m}^{-1}$ given by relativistic equation (2). We have marked in bold the lines of table 1 , corresponding to rotation frequencies 11000 , 


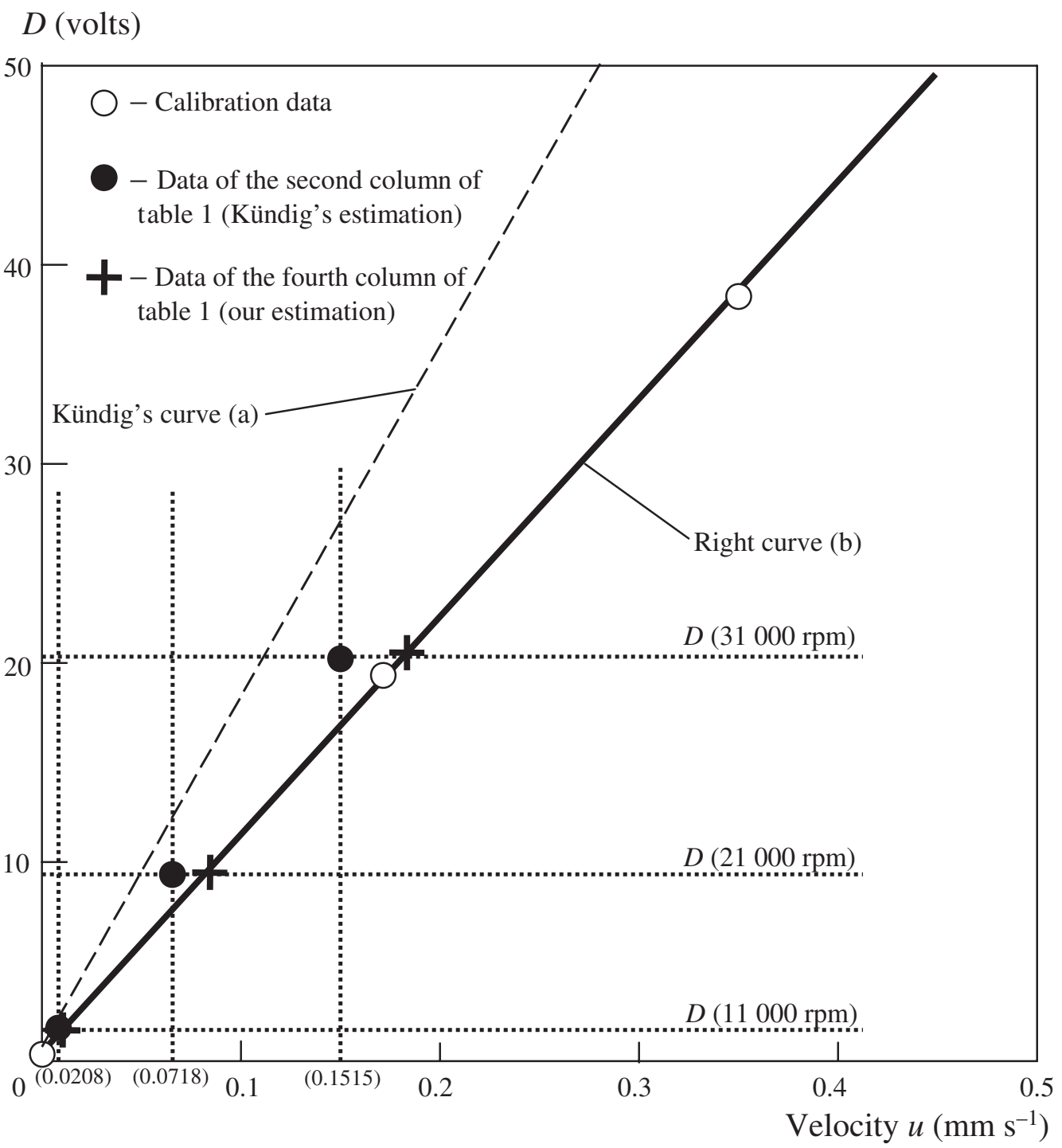

Figure 2. Calibration curves plotted by Kündig (a) and the present authors (b) in comparison with calibration data (white circles) and the data of table 1.

Table 1. The results of Kündig's experiment (columns 1-3) in comparison with the present authors' estimations (column 4).

\begin{tabular}{lccc}
\hline $\begin{array}{l}\text { Speed of rotor } \\
\text { (rpm) }\end{array}$ & $\begin{array}{c}\text { Shift } D\left(10^{-6} \mathrm{~ms}^{-1}\right) \\
(\text { Kündig) }\end{array}$ & $\begin{array}{c}D /\left(R_{\mathrm{A}}^{2}-R_{\mathrm{s}}^{2}\right) \omega^{2}\left(10^{-9} \mathrm{sm}^{-1}\right) \\
(\text { Kündig) }\end{array}$ & $\begin{array}{c}D /\left(R_{\mathrm{A}}^{2}-R_{\mathrm{s}}^{2}\right) \omega^{2}\left(10^{-9} \mathrm{sm}^{-1}\right) \\
\text { (our estimation) }\end{array}$ \\
\hline 3000 & $-1.5 \pm 1.8$ & $-1.7 \pm 2.1$ & - \\
$\mathbf{1 1 ~ 0 0 0}$ & $\mathbf{2 0 . 8} \pm \mathbf{1 . 5}$ & $\mathbf{1 . 8 0 3} \pm \mathbf{0 . 1 2 7}$ & $\mathbf{1 . 9 6 5} \pm \mathbf{0 . 1 1}$ \\
$\mathbf{2 1 ~ 0 0 0}$ & $\mathbf{7 1 . 8} \pm \mathbf{1 . 2}$ & $\mathbf{1 . 7 0 5} \pm \mathbf{0 . 0 2 9}$ & $\mathbf{1 . 9 5 5} \pm \mathbf{0 . 0 2 5}$ \\
25000 & $101.4 \pm 1.5$ & $1.703 \pm 0.026$ & - \\
$\mathbf{3 1 ~ 0 0 0}$ & $\mathbf{1 5 1 . 5} \pm \mathbf{2 . 3}$ & $\mathbf{1 . 6 5 3} \pm \mathbf{0 . 0 2 5}$ & $\mathbf{2 . 0 3 7} \pm \mathbf{0 . 0 2 0}$ \\
35000 & $195.0 \pm 2.3$ & $1.666 \pm 0.020$ & - \\
Weighted average & & $1.679 \pm 0.013$ & $1.986 \pm 0.01$ \\
Expected result $=1 / 2 c$ & & 1.668 & $?$ \\
\hline
\end{tabular}

21000 and $31000 \mathrm{rpm}$, for which the original experimental data were presented by Kündig in [2] and hence which can be evaluated independently. The data of the second column of table 1 marked in bold are shown in figure 2 as black cycles. One can see that they are not compatible with Kündig's calibration curve (a) or with our own calibration curve (b). Thus the origin of these data remains unclear. Our own estimation of the shift $D$ (in velocity units) with the calibration curve (b) of figure 2 gives the values shown in the fourth column of table 1 and depicted in figure 2 by crosses.
Thus, we reveal a valuable discrepancy with the results reported by Kündig. In particular, the weighted average of $D /\left(R_{\mathrm{A}}^{2}-R_{\mathrm{s}}^{2}\right) \omega^{2}$ is equal to $(1.986 \pm 0.01) \times 10^{-9} \mathrm{~s} \mathrm{~m}^{-1}$, and is approximately $20 \%$ higher than Kündig's result, $(1.679 \pm 0.013) \times 10^{-9} \mathrm{~s} \mathrm{~m}^{-1}$. Correspondingly, instead of the relative energy shift (1) estimated by Kündig, we obtain

$$
\frac{\Delta E}{E}=-(1.192 \pm 0.011) \frac{R_{\mathrm{A}}^{2} \omega^{2}}{2 c^{2}} .
$$



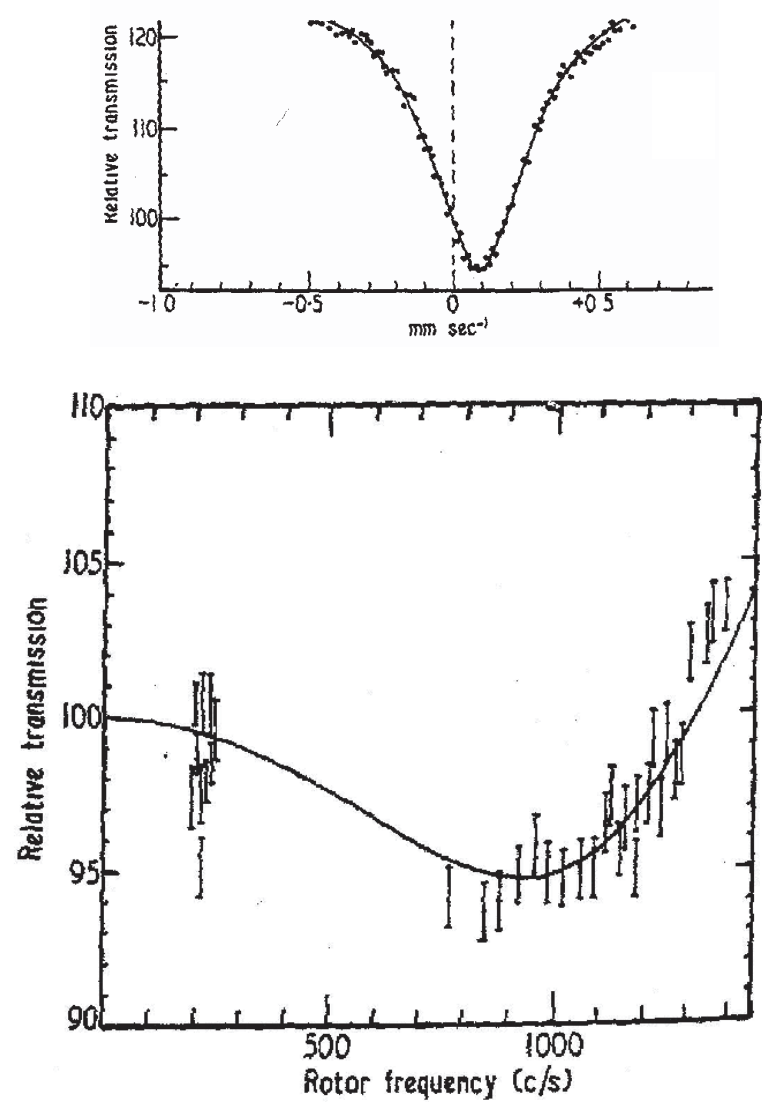

Figure 3. (a) Mössbauer spectrum of the absorber $\mathrm{K}_{4} \mathrm{Fe}(\mathrm{CN})_{6}$, obtained with the source ${ }^{57} \mathrm{Co}(\mathrm{Cr})$. (b) Relative transmission of this absorber in the rotor experiment

\section{Other Mössbauer experiments on the transverse Doppler shift}

We would like to emphasize again that due to applied modulation of energy of emitting resonant radiation, Kündig was successful in measuring a position of the resonant line on the velocity (energy) scale, which is almost insensitive to vibrations of the rotor. This methodological feature favorably distinguishes his experiment from others mentioned above [3-7], where a possible influence of chaotic vibrations on the width of the resonant line in fact was ignored. It is worth pointing out that Kündig observed an increase in linewidth by more than 1.5 times under an increase in the rotation frequency from 11000 to $31000 \mathrm{rpm}$. This does not mean yet that the same appreciable variation of linewidth took place for the rotors applied in [3-7]. At the same time, it is rather difficult to believe that a variation of linewidth was totally absent, as assumed by the authors of the mentioned papers [3-7]. Among them, the experiment by Champeney et al [7] is distinguished by the numerous experimental data, obtained for different absorbers (five pieces) and Mössbauer sources, ${ }^{57} \mathrm{Co}$, in two different matrices. At the same time, only for the source ${ }^{57} \mathrm{Co}(\mathrm{Cr})$ and the absorber $\mathrm{K}_{4} \mathrm{Fe}(\mathrm{CN})_{6}$ do the authors represent simultaneously the Mössbauer spectrum (figure 3(a), which can be considered a calibration measurement) and the result of the rotor experiment (figure 3(b)). Thus, only for this combination can the reader independently verify the results obtained.

One can see that the statistic quality of the rotor experiment [7] (figure 3(b)) is not high. Nevertheless, the authors of [7] were successful in drawing an approximating curve (continuous line) and in estimating the relative energy shift averaging over 16 runs as

$$
\frac{\Delta E}{E}=-(1.02 \pm 0.021) \frac{v^{2}}{2 c^{2}}
$$

in full agreement with the expected relativistic prediction.

However, we pay attention to three groups of experimental points lying outside the approximating curve. The first (left) group corresponds to the rotational frequency near $200 \mathrm{cs}^{-1}$ and apparently reflects an unstable operation of the rotor at these comparably low frequencies, which is accompanied by a variable level of vibration. It is more interesting to explain the deviation of the central (near $800 \mathrm{cs}^{-1}$ ) and right (1300-1400 $\mathrm{cs}^{-1}$ ) groups of experimental points. We assume that the right group of points obtained at extremely high frequencies $1300 \mathrm{cs}^{-1}$ reflects a known effect of reducing the chaotic vibrations in a rotor, when a centripetal acceleration approaches the strengthen limit of the rotor's material. If so, the experimental points at the frequency range $\sim 900-1300 \mathrm{cs}^{-1}$ should also lie higher than the approximating continuous curve in the absence of vibrations. For the assumed uncertainty it seems especially important to determine an exact position of a minimum of the approximating curve for data in figure 3(b), which is essentially less sensitive to rotor vibration than the shape of this curve. The approximating line drawing by Champeney et al [7] gives a minimum at a frequency of about $950 \mathrm{cs}^{-1}$. Now we pay attention to the central group of experimental points, which lie below the approximating curve and allow us to suppose that the actual extreme is located at the frequency $800-830 \mathrm{cs}^{-1}$. Using the calibration curve in figure 3(a) and drawing an approximating curve $\Delta E / E=-\lambda v^{2} / 2 c^{2}$ ( $\lambda$ being the variable parameter) with the minimum at $\sim 800 \mathrm{cs}^{-1}$, we found that this curve also passes through the experimental data points of $v=1300-1400 \mathrm{cs}^{-1}$. As a result, we get the estimation

$$
\frac{\Delta E}{E}=-(1.21 \pm 0.050) \frac{v^{2}}{2 c^{2}},
$$

which agrees with our result (5) derived from Kündig's experimental data. We do not insist that the estimation (6) exactly follows from the Champeney et al experiment. Rather we wanted to demonstrate that this experiment, like the other above mentioned rotor experiments without energy modulation of resonant gamma-quanta, bears an ambiguous interpretation. In these conditions, we may consider the Kündig experiment (were the related data appropriately treated) as the most reliable one for the measurement of a relative energy shift between the resting resonant source and the rotating resonant absorber.

\section{Discussion}

Now we ask the crucial question on the origin of the deviation of Kündig's result from relativistic prediction on the time 
dilation effect. We trust the validity of the usual relativistic dilation of time due to the motion, which, as we mentioned above, has numerous confirmations in the experiments dealing with atomic beams and free muons ([8,9] and references therein). Rather we conjecture that in the Kündig experiment, the energy shift of absorption resonant line is induced not only by the standard time dilation effect, but also by some additional effect missed at the moment. Equation (5) shows that this additional relative energy shift has the order of magnitude

$$
\frac{\Delta E}{E} \approx-0.2 \frac{v^{2}}{2 c^{2}}=-0.1 \frac{v^{2}}{c^{2}}
$$

and, for the rotation frequency $31000 \mathrm{rpm}$, it reaches the value of

$$
\Delta E / E \approx-10^{-13}=0.15 \Gamma,
$$

$\Gamma$ being the natural linewidth of ${ }^{57} \mathrm{Fe}$ resonance.

In order to clarify the possible nature of the energy shift (8), we point out that in the rotor experiments a receiver of radiation (resonant absorber) experiences a centrifugal force $\vec{F}$, compensated by mechanical stresses in the sample holder. This force creates a pressure $p$ in the absorber at the value

$$
p=\frac{F}{S}=\frac{m_{\mathrm{A}} \omega^{2} R_{\mathrm{A}}}{S}=\rho l \omega^{2} R_{\mathrm{A}},
$$

where $S$ is the surface area of absorber, and $m_{\mathrm{A}}, \rho, l$ are its mass, density and thickness, correspondingly. The pressure can influence hyperfine fields in $\alpha$-iron absorber and, correspondingly, a position of the absorption line. However, even for the highest rotational frequency in Kündig's experiment (35000 rpm), and $\rho=7.9 \mathrm{~g} \mathrm{~cm}^{-3}$ (iron), $l \approx 10 \mu \mathrm{m}$, the pressure $p$ in equation (9) does not exceed 1 bar, whereas detectable variations of resonant lines are observed beginning with the pressure of a few kilobars ([10] and references therein). On the other hand, the absorber can experience not only a pressure due to its centrifugal force, but also partially the pressure of its holder. In such a case, the pressure can be essentially increased, and it depends on the holder's mass and its construction. In particular, our estimation shows that for the holder's mass 5-10g, the effective maximal pressure could be equal to $\approx 1 \mathrm{kbar}$. However, even in this case, a corresponding change of electron density on resonant nuclei of the absorber induced by a pressure seems insufficient to explain the additional energy shift (7). Thus, other possible explanations for the revealed effect are very much required. We suppose that such an explanation should take into account not only the influence of pressure but also the above mentioned physical difference between the free ion beam (where the time dilation effect was measured in 'a pure form') and resonant nuclei in the rotating absorber, which represent a quantum system. Besides, one should mention that this quantum system, from a viewpoint of observer in a rotating frame, is in an effective gravitational field. Although the general relativity also leads to equation (2) for such a rotating observer, one of the co-authors of the present contribution suggested an alternative approach, leading to the appearance of an extra-shift of photon's energy in comparison with equation (2) $[11,12]$. Not going into the details of Yarman's analysis, which is obviously outside the scope of the present paper, we nevertheless recognize that only Yarman's hypotheses motivated us to re-examine Kündig's experiment.

\section{Conclusion}

We emphasize a principal result of our analysis: at the moment there is only one reliable Mössbauer experiment for the measurement of the time dilation effect in a rotating frame (Kündig's experiment) and it certainly indicates a deviation from the standard relativistic prediction. We are inclined to think that the revealed deviation (of about 20\%) cannot be explained by any instrumental error. Thus, we hope to stimulate further theoretical and experimental activity, in order to understand the origin of the revealed effect. We were sad to know that Walter Kündig died 2 years ago. We give him due merit for the realization of the ingenious experiment, representing one of the first fundamental applications of the then recently discovered Mössbauer effect, when the methodology of Mössbauer spectroscopy was in its infancy. We seem not to be able to know the required technical details of this experiment with respect to the absorber's holder and some others. Thus, we have decided to repeat Kündig's experiment with the application of recent methodological achievements of Mössbauer spectroscopy described, for example, in $[13,14]$. We will report the results obtained in the due course of time.

\section{References}

[1] Ives H E and Stilwell G R 1938 J. Opt. Soc. Am. 28215

[2] Kündig W 1963 Phys. Rev. 1292371

[3] Hay H J et al 1960 Phys. Rev. Lett. 4165

[4] Hay H J 1962 Proc. 2nd Conf. on the Mössbauer Effect ed A Schoen and D M T Compton (New York: Wiley) p 225

[5] Granshaw T E and Hay H J 1963 Proc. Int. School of Physics, 'Enrico Fermi' (New York: Academic Press) p 220

[6] Champeney D C and Moon P B 1961 Proc. Phys. Soc. 77350

[7] Champeney D C, Isaak G R and Khan A M 1965 Proc. Phys. Soc. 85583

[8] McGowan R W, Giltner D M, Sternberg S J and Lee S 1993 Phys. Rev. Lett. 70251

[9] Bailey I et al 1977 Nature 268301

[10] Pasternak M P and Taylor R D 1999 Mössbauer Spectroscopy in Material Science ed M Miglierini and D Petridis (Dordrecht: Kulwer) pp 349-58

[11] Yarman T 2005 Proc. Int. Meeting on Physical Interpretation of Realtivity Theory (Moscow, 4-7 July 2005) ed M C Duffy et al (Moscow: BMSTU PH)

[12] Yarman T, Rozanov VB and Arik M 2007 Proc. Int. Meeting on Physical Interpretation of Realtivity Theory (Moscow, 2-5 July 2007) ed M C Duffy et al (Moscow: BMSTU PH)

[13] Evdokimov V A et al 1995 Nucl. Instrum. Methods B 95278

[14] Kholmetskii A L, Evdokimov V A, Mashlan M and Missevitch O V 2004 Hyperfine Interact. 156/157 3 